\title{
ARTICLE
}

\section{Sorption parameter setting approaches for radioactive waste disposal considering perturbation effects: sorption reduction factors for organics}

\author{
Yukio Tachi $^{\mathrm{a}^{*}}$ and Michael Ochs ${ }^{\mathrm{b}}$ \\ ${ }^{a}$ Japan Atomic Energy Agency (JAEA), 4-33 Muramatsu, Tokai, Ibaraki, 319-1194, Japan; ${ }^{b}$ Arcadis Switzerland Ltd, Ifangstrasse 11, \\ CH-8952 Schlieren/Zürich, Switzerland
}

\begin{abstract}
Various types of post-accident radioactive waste have been generated from cleanup and decommissioning activities at the Fukushima Daiichi nuclear power plant. Perturbation effects resulting from co-existing substances may influence radionuclide sorption. For the disposal of these wastes, perturbation effects on sorption parameters must be critically evaluated for the safety assessment of the disposal systems. The present study focuses on developing a methodology to quantify sorption parameters in the presence of such perturbation effects and on illustrating example calculations regarding sorption reduction factors (SRFs) due to the presence of organic ligands (ISA; isosaccharinic acid) for cement systems. Three approaches for the derivation of SRFs; 1) semi-quantitative estimation based on analogy with solubility enhancement factors (SEFs) derived from measured solubility, 2) prediction based on radionuclide speciation calculation, and 3) quantification from experimental sorption data in ternary systems were coupled and tested for the Am-ISA-cement system. Our approach allows to critically evaluate the dependence of sorption reduction factors for various perturbed systems on the chosen method of quantification, in accordance with the data availability for a given perturbation.
\end{abstract}

Keywords: post-accident radioactive waste; Fukushima Daiichi nuclear power plant; perturbation effect; sorption reduction factor; organic ligands; ISA; Am; cement

\section{Introduction}

Following the accident at the Fukushima Daiichi (1F) nuclear power plant (NPP) in March 2011, various types of post-accident radioactive waste have been generated from the cleanup and decommissioning activities (e.g. treatment of contaminated water and fuel debris) [1]. These radioactive wastes have different properties compared with wastes generated by decommissioning of NPPs under normal conditions [1]. For the disposal of these wastes from the $1 \mathrm{~F}$ NPP, perturbation effects resulting from co-existing substances (e.g., organic ligands, borate, and salts) need to be considered. Such co-existing substances may influence radionuclide (RN) transport through the complexation with RNs and competition with RN sorption, and need to be considered in the setting of parameter for the safety assessment of the disposal systems. The retardation of $\mathrm{RN}$ by sorption is a central safety feature of all types of disposal systems. Depending on the estimated impact of complexing chemicals on the safety performance, it may be required to limit the concentration of such co-existing substances in the waste. The big challenge here is that such evaluations must be done for post-accident

*Corresponding author. Email: tachi.yukio@jaea.go.jp radioactive wastes with chemical complexity and insufficient characterization. This situation requires a sound understanding of the pros and cons of different estimation methods.

The present study focuses on developing a methodology to quantify sorption parameters based on sorption reduction factors (SRFs) in the presence of perturbation effects by coupling different approaches depending on data availability. For the present purpose of demonstrating the different estimation methods, isosaccharinic acid (ISA) resulting from cellulose degradation was selected. In the absence of specific information on the organics present in post-accident wastes, ISA is a good candidate for a worst-case evaluation due to its significant effects on complexation with RNs (especially actinides [2,3]) and on the reduction of RNs sorption [4-6].

\section{Generic approaches for handling sorption reduction by perturbation effects}

For safety assessment calculations, the magnitude of sorption is normally expressed by a distribution coefficient $\left(\mathrm{K}_{\mathrm{d}}\right)$. $\mathrm{K}_{\mathrm{d}}$ depends critically on relevant geochemical baseline conditions and additionally on perturbations from co-existing substances (e.g., organic 
ligands, borate, and salts). To treat sorption reduction considering the various perturbations, several options exist. These options range from using pragmatic sorption reduction factors (SRF) to the application of thermodynamic sorption models (TSMs). SRFs are a pragmatic way of representing the decrease of $\mathrm{RN}$ sorption as a function of a perturbation. SRFs quantify the reduction of $\mathrm{RN}$ uptake in the presence of organic and inorganic ligands with respect to the uptake in such ligands-free system [e.g., 6]. The $\mathrm{K}_{\mathrm{d}}$ under perturbed conditions $\left(\mathrm{K}_{\mathrm{d} \text {, perturbed }}\right)$ can be expressed by the baseline $\mathrm{K}_{\mathrm{d} \text {, baseline }}$ in the absence of perturbations and the sorption reduction factor (SRF):

$$
\mathrm{K}_{\mathrm{d} \text {, perturbed }}=\mathrm{K}_{\mathrm{d} \text {, baseline }} / \mathrm{SRF}
$$

Similar to $K_{d}$, SRF are useful for systems with given constant conditions. A typical application is to quantify the influence of complexing ligands on $\mathrm{RN}$ sorption in cement systems [4-6]. In cases where perturbations change the overall system geochemistry, the $K_{d}$ data setting exercise has to be completely repeated to include the new sets of conditions. As explained in more detail below, which of these two cases will occur depends of course on the nature of the perturbations, but also on the general variability of conditions in the system of interest.

\subsection{Direct effects / constant conditions (overall system not affected by perturbations)}

In this model case, the perturbations are only relevant with respect to the $\mathrm{RN}$ behavior, but do not affect the overall chemical conditions. This situation can be illustrated by the left-hand side of Figure 1, which can be thought to represent e.g. a cement system. In such a system, the $\mathrm{pH}$ and solution composition are well buffered, and the addition of organic ligands influences mainly the RN behavior. In such a case, the baseline $\mathrm{K}_{\mathrm{d}}$, baseline in the absence of organics is well defined and the $\mathrm{K}_{\mathrm{d}}$ under perturbed conditions $\left(\mathrm{K}_{\mathrm{d} \text {, perturbed }}\right)$ depends only on the organic ligand concentration. Such a change can be well handled by SRFs. In such cases, the influence on sorption can be directly determined in experiments, using the specific perturbing agents and relevant sorbing solids. In case of ligands that are well defined or have at least a constant composition, it is possible to quantify their influence through SRFs. SRFs are conditional, i.e., they are valid for a given set of conditions in the same way as $\mathrm{K}_{\mathrm{d}}$ values.

\subsection{Indirect effects / variable conditions (overall system chemistry is affected by perturbation)}

In this case, the perturbations do not only affect RN behavior, but also some or all geochemical conditions of the system. In a simple case, the $\mathrm{pH}$ may vary in addition to the concentration of organics. In such a case, a simple approach like SRF cannot be applied, instead a more comprehensive approach should be used as shown in the right-hand side of Figure 1. For example, a change from a given system's $\mathrm{pH}$ to highly alkaline conditions would induce a number of other changes. Such cases, where several parameters are changed at the same time, are often treated like two separate, independent systems. I.e., changing a system to highly alkaline conditions may likely require an independent data setting.

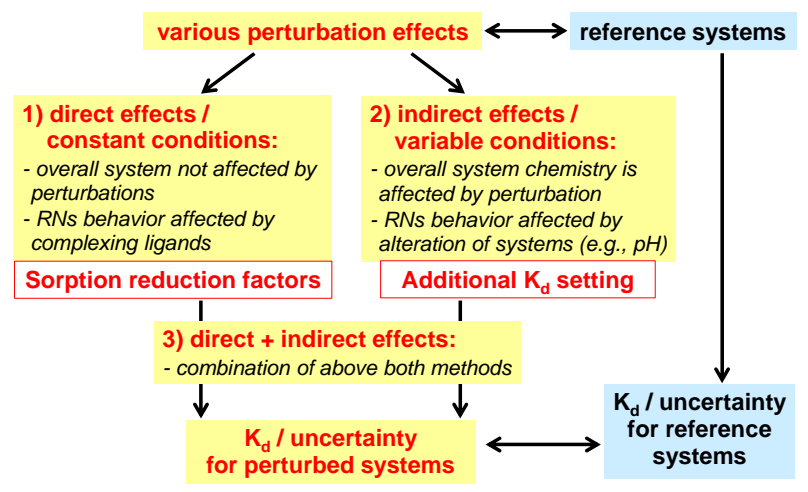

Figure 1. Simplified overview of how perturbations and sorption reduction effects may be addressed in different situations.

3. Parameter setting approaches for sorption reduction factors (SRFs): Illustration for organic substances

In the following, sorption parameter setting based on SRF in the case of above-mentioned direct effects / constant conditions is illustrated. Discussion of a system with variable conditions is outside the scope of this paper.

\subsection{Target system for deriving SRFs}

In general, SRFs appear to be mainly used in the context of $\mathrm{K}_{\mathrm{d}}$ setting for cement systems containing complexing ligands [4-6]. There are two main reasons for this observation: First, cement systems are relatively well buffered in terms of $\mathrm{pH}$ and other solution parameters, so that the presence of additional substances (such as organic ligands) affects only RN sorption, but not $\mathrm{pH}$ and other main parameters. Second, organic complexing agents are a typical constituent of wastes disposed in cement-based facilities. The present illustration of 3 different methods of SRF derivation focuses on the effect of ISA perturbation on Am sorption in slightly degraded cement systems (region II; $\mathrm{pH} \sim 12.5)[7,8]$.

\subsection{SRF setting based on analogy between solubility enhancement factors (SEF) and SRF (Method 1)}

To our knowledge, the first explicit use of RN sorption reduction factors (SRFs) was made by [4] to quantify the effect of ISA on RN sorption in the context of the first $\mathrm{K}_{\mathrm{d}}$-setting for the Swiss ILW/LLW repository. At the time of their report, relatively few experimental data on the interaction of RNs with ISA were available. It is reported that sorption data in the presence of ISA could only be found for Pu. SRFs for other RNs were 
estimated by assuming analogies between solubility enhancement and sorption reduction, as follows:

- Based on experimental Pu solubility data recorded as a function of ISA concentration at relevant conditions (pH 12), a solubility enhancement factor is derived (tetravalent $\mathrm{Pu}$ is assumed).

- Assuming analogy between solubility enhancement and sorption reduction, realistic sorption mechanisms are pessimistically ignored (e.g., sorption of ISA, formation of ternary surface species, competition by Ca-ions).

- Assuming analogy between Pu(IV) and other actinides, the solubility enhancement - sorption reduction analogy is applied to the other RNs.

\subsection{SRF prediction based on aqueous speciation calculations (Method 2)}

A semi-quantitative $\mathrm{K}_{\mathrm{d}}$ estimation procedure has been developed by considering transferring factors accounting for differences in sorption capacity and surface as well as solution chemistry in the experimental vs. application conditions [9,10]. The complexation of RNs with inorganic and organic ligands has an impact on sorption, therefore, a $K_{d}$ transferring factor for $\mathrm{RN}$ speciation is included in this approach.

When RN speciation calculations are available based on a reliable thermodynamic database (TDB) [2,3], SRFs can be evaluated by considering the formation of RN-ISA complexes as being competitive with regard to sorption. SRFs can be derived using the total dissolved $\mathrm{RN}$ concentration $\left(\mathrm{RN}_{\text {tot }}\right)$ and the total concentration of dissolved RN-ISA complexes $\left(\mathrm{RN}_{\mathrm{ISA}}\right)$ :

$$
\mathrm{SRF}=\mathrm{RN}_{\text {tot }} /\left(\mathrm{RN}_{\text {tot }}-\mathrm{RN}_{\mathrm{ISA}}\right)
$$

The Am speciation calculations in cement porewater at region II (pH 12.5) of cement degradation [7] presented in Figure 2, indicates that Am-ISA complexation (a mixed hydroxo-ISA complex; $\left.\mathrm{Am}(\mathrm{OH})_{3} \mathrm{ISA}^{-}\right)$increases gradually as ISA concentration increases, and that Am speciation is entirely dominated by this ISA complex at higher ISA concentration. As a result, the SRF increases as ISA concentration increases.

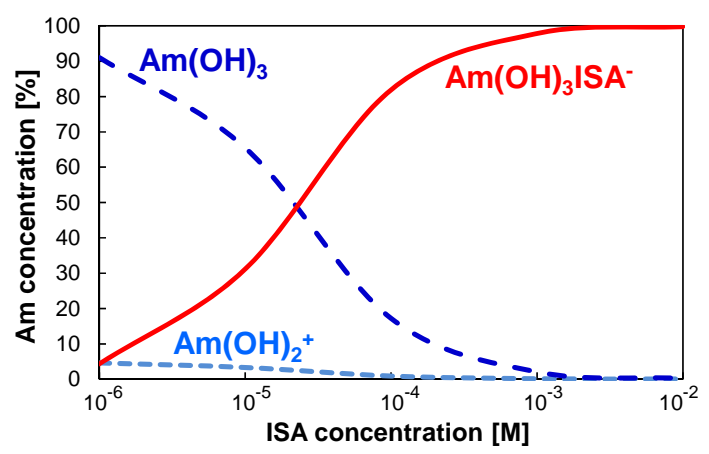

Figure 2. Aqueous Am speciation as a function of ISA concentration, calculated by using the TDB of [3].

\subsection{SRF derivation from experimental sorption data in ternary systems (Method 3)}

The influence on sorption can be directly determined in experiments, using the specific perturbing agents and sorbing solids. In case of ligands that are well defined or have at least a constant composition, it is possible to quantify their influence through SRFs. The SRFs are conditional, i.e., they are valid for a given set of conditions in the same way as $K_{d}$ values. A typical example of the derivation of SRF in a system with constant conditions is shown in Figure 3. This figure illustrates the relation of sorption and ligand concentration at a constant $\mathrm{pH}(\mathrm{pH} 12.5)$, and shows the transition from a ligand concentration range where no effects can be observed to a concentration range where sorption decreases strongly and approximately linearly with increasing ligand concentration.

- The no-effect level of $1.0 \times 10^{-4} \mathrm{M}$ is based on direct observation, giving more weight to the data obtained by [5], from a conservative point of view. The selected no-effect level takes into account the uncertainty in the baseline $\mathrm{K}_{\mathrm{d}}$ values.

- SRF was selected as a 10-fold decrease in sorption with each 10-fold increase in ISA concentration, representing the slope of the sorption data.

This example shows that even under otherwise constant conditions, SRF usually have to be defined as a function of ligand concentration. In many situations, a single SRF is only valid for a narrow range of complexing ligand concentration. In many systems with constant composition it may similarly be possible to investigate the effect of increased salinity (or other salts, such as borates) without disturbing other system parameters too much. This would allow a similar treatment as in case of additional complexants; i.e., using no-effect concentrations and SRFs.

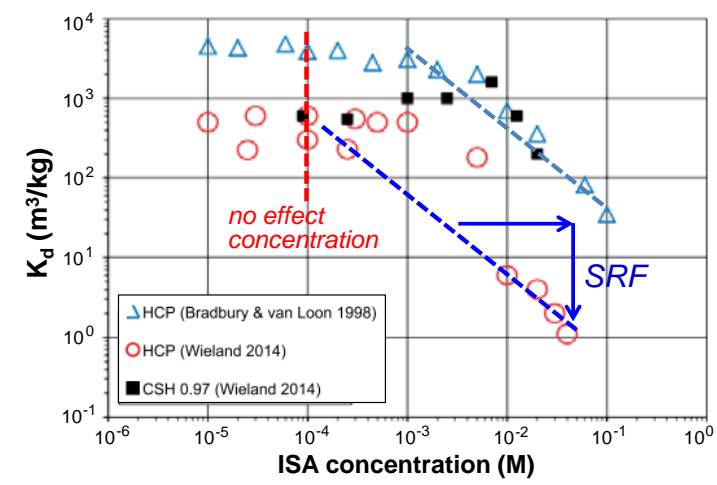

Figure 3. Illustration of the relations between $\mathrm{RN}$ sorption $\left(\mathrm{K}_{\mathrm{d}}\right)$ and ISA concentration in a cementitious environment at $\mathrm{pH}=$ 12.5 (modified from [6]). The illustrated case shows the uptake of Eu by hydrated cement paste (HCP) and a Ca-silicate-hydrate (CSH) in the presence of ISA $[5,11]$.

\subsection{Discussion: Comparison of SRFs from different methods}

SRFs for the cement-Am-ISA case obtained by methods (1)-(3) are compared in Figure 4. The SRF 
trends as a function of ISA concentration estimated by the three methods explained above are qualitatively consistent; however, their advantages and drawbacks can be summarized as the followings :

- Method (1): SRFs derived from solubility data are not necessarily good analog measures, leading to a significant overestimation of SRFs. Of course, if nothing else is available, solubility data are the best starting point.

- Method (2): SRF estimation based on RN speciation appears as a better option. By neglecting e.g. sorption of ISA and formation of ternary surface species, it is likely to overestimate SRFs. This method also depends on the quality of the TDB used so this has to be handled carefully.

- Method (3): Reliable SRFs can be derived directly from experimental data for ternary systems, i.e, where the effect can be directly measured in the presence of solid surface, RN and ligand.

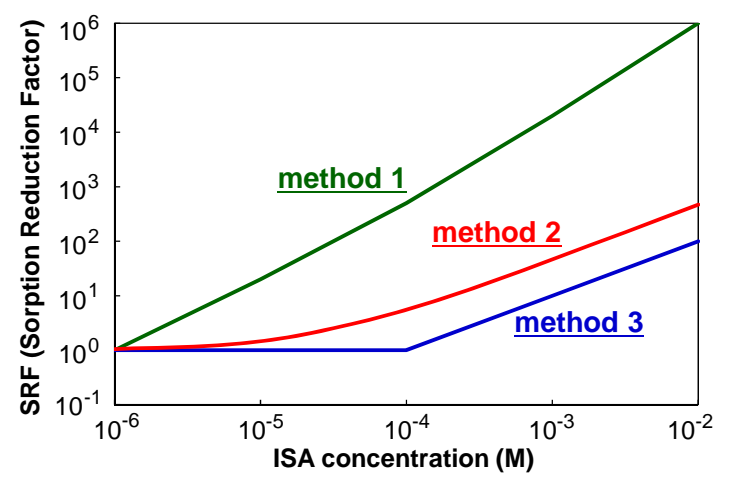

Figure 4. Comparison of SRFs derived by three different methods : 1) analogy between SEF and SRF, 2) RN speciation calculation, and 3) experimental sorption data in ternary system for Am-ISA-cement system.

It can be evaluated that all methods are valid and have certain advantages and drawbacks. Of course, SRFs derived from ternary systems (method 3), i.e, where the effect can be directly measured in the presence of solid surface, $\mathrm{RN}$ and ligand, are most reliable. However, SRF often need to be derived in the absence of such direct evidence, and estimations need to be made based on analogies (method 1) and speciation information (method 2). Such estimates may be subject to substantial uncertainties. These should not be seen as something that does not allow to use SRFs, but need to be clearly acknowledged and the SRFs should reflect such uncertainties e.g. by using conservative values or by providing additional conservative estimates.

In very general terms, approaches are ideally selected based on a clearly defined goal, and on the advantages and drawbacks of each method. Which approach will be selected for handling sorption reduction effects that may result from the mentioned system perturbations will of course depend on the available data as well as on the available resources and time.

\section{Conclusion}

A methodology for handling sorption reduction effects resulting from various perturbations was developed by focusing on the method of using sorption reduction factors (SRFs). Three options for deriving SRFs were applied and compared for the Am-ISA-cement system. These SRF approaches are effective when used carefully and can be applied in a stepwise manner according to the data availability for given perturbations.

\section{References}

[1] OECD/NEA, Management of Radioactive Waste after a Nuclear Power Plant Accident, (2016).

[2] X. Gaona, V. Montoya, E. Colàs, M. Grivé and L. Duro, Review of the complexation of tetravalent actinides by ISA and gluconate under alkaline to hyperalkaline conditions, J. Contam. Hydol., 102 (2008), pp. 217-227.

[3] D. Rai and A. Kitamura, Thermodynamic equilibrium constants for important isosaccharinate reactions: A review, J. Chem. Thermodynamics, 114 (2017), pp. 135-143.

[4] M.H. Bradbury, and F.-A. Sarott, Sorption database for the cementitious near-field of a L/ILW repository for performance assessment, Nagra TR 93-08 (1994).

[5] E. Wieland, Sorption data base for the cementitious near field of L/ILW and ILW repositories for provisional safety analyses for SGT-E2, Nagra NTB 14-08 (2014).

[6] M. Ochs, E. Colàs, M. Grivé, J. Olmeda, I., Campos and J. Bruno, Reduction of radionuclide uptake in hydrated cement systems by organic complexing agents: Selection of reduction factors and speciation calculations, SKB R-14-22 (2014).

[7] M. Mihara and R. Sasaki, Radionuclides Migration Datasets (RAMDA) on cement, JNC TN8400 2005-027 (2005).

[8] M. Ochs, B. Vriens and Y. Tachi, Retention of uranium in cement systems: Effects of cement degradation and complexing ligands, Prog. Nucl. Sci. Technol. 5 (2018), pp. 208-212.

[9] M.H. Bradbury and B. Baeyens, Far field sorption data bases for performance assessment of a high-level radioactive waste repository in an undisturbed Opalinus Clay host rock, PSI Bericht Nr.03-08 (2003).

[10]M. Ochs, Y. Tachi, D. Trudel and T. Suyama, $K_{d}$ setting approaches for Horonobe mudstone systems: Applications of TSMs and semiquantitative estimation procedures, JAEAResearch 2012-044 (2013).

[11]M.H. Bradbury and L. Van Loon, Cementitious near-field sorption data bases for performance assessment of a L/ILW disposal facility in a Palfris Marl host rock CEM-94: UPDATE I, Nagra NTB 96-04 (1998). 